\title{
LUCES Y SOMBRAS DE LA DIFICULTAD CONTRAMAYORITARIA
}

\author{
RODRIGO BRITO MELgarejo ${ }^{1}$
}

RESUMEN: En este artículo se hace una revisión de algunas objeciones que se han planteado respecto a la actuación contramayoritaria de los órganos jurisdiccionales de control y se señalan las respuestas que se han dado a cada una de ellas. Esto con el fin de demostrar que, más que dificultades, la forma en que los jueces constitucionales llevan a cabo sus funciones en los sistemas democráticos, ofrece oportunidades para ponderar valorativamente la práctica jurídico-institucional vigente en un Estado, para generar equilibrios entre los órganos de gobierno y la sociedad, así como para lograr mejores escenarios deliberativos.

Palabras clave: Control jurisdiccional de constitucionalidad, democracia, dificultad contramayoritaria, sistemas de control.

ABSTRACT: This article aims to make a revision of some objections that have been raised about the countermajoritarian actuation of judges and indicate the answers that have been given to each of them. With the purpose to demonstrate that, rather than difficulties, the way in which constitutional judges perform their functions in democratic systems, offers opportunities to evaluate the current legal-institutional practice in a State, to generate balance between governing and society, as well as to achieve better deliberative scenarios.

KEYWORDS: Fudicial review, democracy, countermajoritarian difficulty.

SumARIO: I. In limine; II. Las objeciones a la actuación contramayoritaria de los órganos jurisdiccionales de control; III. La oportunidad contramayoritaria; IV. A manera de conclusiones.

1 Profesor de la Facultad de Derecho de la UNAM y doctor en justicia constitucional y derechos fundamentales por la Università degli Studi di Pisa: <rbritom@derecho.unam.mx>. 


\section{IN LIMINE}

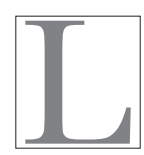

as tensiones entre la democracia y el control jurisdiccional de constitucionalidad han sido abordadas por la doctrina desde diversos puntos de vista. Para algunos, esas tensiones ofrecen oportunidades que favorecen a los Estados constitucionales; mientras que para otros, el que los órganos de control realicen sus funciones revisando las decisiones que toman ciertos poderes estatales que cuentan con legitimidad democrática, atenta contra la soberanía popular y, por tanto, contra ciertas preferencias que deberían respetarse. El debate que se presenta a partir de estas posiciones contrapuestas, permite encontrar luces y sombras respecto a una figura que ha cobrado especial importancia en los últimos años dentro del debate constitucional: la dificultad contramayoritaria. Esta expresión, acuñada por Alexander Bickel, ${ }^{2}$ se utiliza cuando se quiere señalar que el control jurisdiccional de la ley es, o al menos parecería ser, una institución diseñada para limitar las decisiones tomadas por los órganos políticos que representan la voluntad popular mayoritaria. ${ }^{3}$ De esta manera, se estaría produciendo un cambio en el paradigma de la toma de decisiones, pasándose de uno "democrático" a otro "elitista", el cual se materializaría en una minoría (los jueces) que toman decisiones que deberían corresponder

2 Bickel, Alexander M., The least dangerous branch: The Supreme Court at the bar of politics, Nueva York, The Bobbs-Merrill Company, 1962.

3 Ferreres Comella, Víctor, Fusticia constitucional y democracia, $2^{\mathrm{a}}$ ed., Madrid, Centro de Estudios Políticos y Constitucionales, 2007, p. 42. Para Bickel, "The root difficulty is that judicial review is a counter-majoritarian force $[\ldots]$ When the Supreme Court declares unconstitutional a legislative act or the action of an elected executive, it thwarts the will of representatives of the actual people of the here and now; it exercises control, not in behalf of the prevailing majority, bus against it. That, without mystic overtones, is what actually happens. It is an altogether different kettle of fish, and it is the reason the charge can be made that judicial review is undemocratic". Bickel, Alexander M., op. cit., pp. 16-17. 
sólo al pueblo y a sus representantes elegidos a través del sufragio. ${ }^{4}$ La idea que subyace en estos planteamientos es que los órganos de control, al no ser elegidos por el pueblo de manera inmediata, no pueden, al controlar la constitucionalidad, ser los censores de poderes del Estado que tienen como respaldo el voto ciudadano. Es por ello que diversos autores han tratado de evidenciar las dificultades que conlleva la esencia contramayoritaria de las decisiones tomadas por el juez constitucional; sin embargo, hay quienes, por el contrario, consideran que la actuación de los órganos de control favorece en buena medida la democracia. A analizar algunas de las objeciones y oportunidades que sobre el actuar contramayoritario de los órganos de control se presentan recurrentemente entre la doctrina se dedican las páginas que siguen.

II. LAS OBJECIONES A LA ACTUACIÓN CONTRAMAYORITARIA DE LOS ÓRGANOS JURISDICCIONALES DE CONTROL

Existen diversas circunstancias que dan pie a la dificultad contramayoritaria. La primera es que la voluntad de la mayoría queda rebasada por unas cuantas personas que forman parte de los órganos jurisdiccionales de control. Esta objeción se ha vinculado con argumentos como los estudiados por Robert Dahl, quien refería que "no amount of tampering with democratic theory can conceal the fact that a system in which the policy preferences of minorities prevail over majorities is at odds with the traditional criteria for distinguishing a democracy from other political systems". ${ }^{5}$ Bajo esta perspectiva, el término democracia implica que el poder de

4 Álvarez Álvarez, Fernando D., "Legitimidad democrática y control judicial de constitucionalidad (Refutaciones al carácter contramayoritario del Poder Judicial)", Revista Díkaion-Lo Justo, año 17, núm. 12, 2003, pp. 151-153.

5 Dahl, Robert A., Decision-making in a democracy: The Supreme Court as a national policy-maker, Fournal of Public Law, vol. 6, 1957, p. 283. 
dirigir reside en las mayorías populares y sus representantes y, por esta razón, afirmar que un órgano de control pueda sostener las preferencias de las minorías frente a las de las mayorías es negar la soberanía popular y la igualdad política. ${ }^{6}$

Entre las circunstancias que favorecen la dificultad contramayoritaria, tiene también un lugar especial el que los órganos de control de constitucionalidad cuenten con una menor legitimidad democrática de origen, pues mientras la ley que deben revisar proviene de un parlamento elegido periódicamente por el electorado a través del sufragio universal, no sucede lo mismo con quienes integran los órganos de control. ${ }^{7}$ La objeción que se sostiene en este sentido es que a través del control jurisdiccional de constitucionalidad la voluntad política de los ciudadanos se frustra. Y es que las personas que desean tomar una determinada decisión política con base en su autoridad democrática, en ocasiones se topan con órganos de control que les dicen que no pueden hacerlo. Este argumento ha estado detrás de las primeras críticas que se registran en la historia respecto al control de constitucionalidad ${ }^{8}$ y encuentra su sustento

6 Idem.

7 Ferreres Comella, Víctor, op. cit., p. 43.

8 Gowder, Paul, "The countermajoritarian complaint", Transnational Law \&̊ Contemporary Problems, vol. 23, núm. 7, 2014, pp. 10-11. Gowder ilustra esta afirmación señalando que "In 406 BC, during the Peloponesian War, Athens won a major sea battle against Sparta, the battle of Arginusae. Unfortunately, a storm hit after the battle, and many of the Athenian sailors were lost on the way home. Enraged at the failure to rescue their fellow citizens from the storm, the Athenian people, acting in their sovereign democratic capacity in the ekklesia (assembly), put the generals on trial en masse and executed them without giving them an adequate opportunity to defend themselves. Essentially, the ekklesia passed an act of attainder against the generals. This mass trial was illegal. One citizen, Eryptolemus, attempted to put a stop to it by bringing a graphe paranomon -an indictment charging the proposers of the mass trial with making an illegal proposal. The indictment would have been tried by a citizen jury, itself a representative of the sovereign authority of the polis, randomly selected 
en la dignidad democrática de la ley, que es consecuencia de su aprobación por el órgano del Estado que está en la mejor posición institucional para expresar la voluntad popular: el parlamento elegido periódicamente por sufragio universal. ${ }^{9}$

En este punto, podría pensarse que la actuación de los órganos de control sería susceptible de superarse a través de la labor de órganos con legitimidad democrática que, eventualmente, estarían en aptitud de realizar las modificaciones constitucionales necesarias para superar las decisiones del controlante. No obstante lo anterior, y más allá de lo criticable que podría ser una determinación como la señalada, en tal caso surgiría otra de las objeciones a la actuación de los órganos que tienen a su cargo el control de constitucionalidad, pues en ocasiones no es fácil modificar los textos constitucionales que, por regla general, están sujetos a un procedimiento de reforma más gravoso que el que se sigue para modificar las leyes ordinarias. En este caso, se supeditaría a lo decidido en el pasado el control de las normas que rigen en el presente a una comunidad política determinada, y ésta, debido a lo difícil que en ocasiones resulta cambiar los textos constitucionales, encontraría complejo revertir una situación de ese tipo. ${ }^{10}$

from ordinary citizens [...] Nonetheless, according to Xenophon, the rest of the citizens responded with outrage at the prospect that Euryptolemus would dare to tell them that they could not exercise their authority in the assembly according to their collective whim: 'the multitude shouted out that it was a terrible thing if someone prevented the people from doing whatever they wished'. Under the threat that he too would be executed by the runaway assembly, Euryptolemus withdrew the writ, and the generals were duly executed".

9 Ferreres Comella, Víctor, op. cit., p. 38.

10 Gowder señala al respecto: "Constitutional law, at least in countries like the United States, is entrenched because of the onerous requirements of the amendment process - such as supermajorities. The majoritarian legislature does not have full prospective control over the laws that regulate its behavior". Gowder, Paul, op. cit., p. 16. 
Aunada a esta dificultad se presenta otra objeción a las facultades contramayoritarias con que cuentan los órganos de control: la competencia que éstos tienen para interpretar la Constitución y, por tanto, para imponer su punto de vista sobre la Constitución por largos periodos. ${ }^{11} \mathrm{Al}$ ser los jueces constitucionales quienes frecuentemente dotan de contenidos a las constituciones y determinan el sentido que debe darse a sus preceptos, éstos pueden imponer su visión sobre el texto constitucional a la sociedad, sin que ésta tenga a su alcance mecanismos que no sean extremos para cambiar sus determinaciones. Esto puede ser especialmente frecuente dada la controvertibilidad interpretativa de la Constitución. Debido a la abundancia en los textos constitucionales de "conceptos esencialmente controvertidos" (principalmente en materia de derechos y libertades) así como a las colisiones entre las diversas disposiciones constitucionales, la interpretación de las normas fundamentales se torna más controversial que la de las leyes ordinarias ${ }^{12} \mathrm{y}$, por ende, ofrece una veta mayor para la actuación de los órganos de control, que pueden imponer la perspectiva que tienen sobre los contenidos constitucionales.

También se ha dicho que el pueblo soberano podría justamente reclamar que tiene la responsabilidad de preservar la moral representada por su propia comunidad política y, consecuentemente, bajo ciertas circunstancias extremas, de tomar en última instancia la decisión de desatender el orden jurídico para lograr tal fin. Asumiendo este planteamiento, la objeción a la actuación contramayoritaria del órgano controlante sería que éste anularía la posibilidad de que el pueblo hiciera suya esta responsabilidad. ${ }^{13}$

11 Ferejohn, John y Pasquale Pasquino, "The countermajoritarian opportunity", University of Pennsylvania Journal of Constitutional Law, vol. 13, núm. 2, 2010-2011, p. 354.

12 Ferreres Comella, Víctor, op. cit., p. 43.

13 Gowder, Paul, op. cit., p. 14. 
Un argumento más que se utiliza para objetar la actuación de los órganos de control en los sistemas democráticos, es que éstos no están sujetos a procesos de fiscalización y responsabilidades en la misma manera en que lo están los órganos representativos. En este sentido se ha señalado que la posibilidad de fiscalizar los actos de los representantes permite a los ciudadanos reemplazar periódicamente a quienes integran, por ejemplo, los órganos legislativos. De esta forma, los poderes que se conforman a través de elecciones son responsables ante el electorado, algo que no sucede con los integrantes de los órganos de control, quienes por regla general no son responsables ni directa ni regularmente ante los ciudadanos. Si bien en muchas ocasiones los jueces constitucionales deben sobrevivir a un proceso mayoritario de nominación y confirmación en el que participan los órganos representativos, es difícil que, una vez electos, existan mecanismos efectivos para hacerlos responsables frente a los ciudadanos y sus represenantes. Los integrantes de los órganos de control, por tanto, no pueden ser reemplazados directamente por el electorado y no están sujetos a la voluntad de los representantes populares. De hecho, se ha dicho que estos servidores públicos raramente asumen los costos de sus errores. ${ }^{14}$ Or Bassok, al referirse a los argumentos de este tipo que usualmente son empleados para sostener la dificultad contramayoritaria refiere que:

The [countermajoritarian]difficulty emphasizes the majoritarian aspect of democracy, i.e. the Court's responsiveness to public opinion. Elected branches are responsive to the majority's will as expressed in elections and in other manifestations such as opinion polls. They adopt policies that are signaled as preferred by citizens. Justices, on the other hand, have lifetime tenure, which relieves them from the need to be responsive to the majority (of the public or its

14 Bassok, Or, "The two countermajoritarian difficulties", Saint Louis University Public Law Review, vol. XXXI, núm. 333, 2011-2012, pp. 339-343. 
representatives), as well as an ethos of unresponsiveness to popular pressure. ${ }^{15}$

Así, la falta de mecanismos a cargo del electorado que pudieran sujetar la actuación de los órganos de control a la opinión pública, se ha esgrimido como uno de los pilares de la dificultad contramayoritaria. Esta objeción evidencia algunas de las particularidades de la relación entre quienes integran los órganos de control y la sociedad, a las que se suman otras críticas derivadas también de dicha relación. Por ejemplo, se ha señalado que a través del control los jueces constitucionales alejan del debate público muchos de los temas cruciales relacionados con los derechos y, de esta forma, su actuación afecta la democracia. De hecho, Mark Tushnet, uno de los autores que adopta este punto de vista, ha referido que "[d] oing away with judicial review would have one clear effect: it would return all constitutional decision-making to the people acting politically. It would make populist constitutional law the only constitutional law there is". ${ }^{16}$ Esta idea ha sido sostenida por otros doctrinarios como Robert Nagel, quien considera que la creencia de que los

15 Idem. Or Bassok se refiere al caso de los fustices de los Estados Unidos y por eso hace mención a su carácter vitalicio; sin embargo, no debe olvidarse que la estabilidad en el cargo es una de las caracterísitcas que garantizan la autonomía e independencia de los integrantes de los órganos de control, por lo que esta afirmación podría extenderse a muchos casos más, en los que los periodos de ejercicio son muy prolongados. Este tema ha sido tratado en Wilson, James G., "The role of public opinion in constitutional interpretation", Brigham Young University Law Review, núm. 4, 1993, p. 1120. En este artículo Wilson señala que "many Justices have separated the judicial domain, excluding public opinion from the political domain, where public opinion reigns supreme".

16 Tushnet, Mark, Taking the Constitution away from the Courts, New Jersey, Princeton University Press, 1999, p. 154. 
jueces puedan confrontar de manera rutinaria a la sociedad y transformarla, evidencia una perspectiva bastante estrecha. ${ }^{17}$

La combinación de estos planteamientos -que pueden presentarse en diversos grados en los distintos sistemas de control- conforman en buena medida el escenario sobre el que se proyectan las sombras que parte importante de la doctrina ve en la actuación contramayoritaria de los órganos controlantes.

\section{LA OPORTUNIDAD CONTRAMAYORITARIA}

Las objeciones que se plantean respecto a la labor contramayoritaria de los órganos de control, han sido abordadas también por estudiosos que ven en ellas argumentos que más que dificultades, ofrecen oportunidades para los sistemas democráticos. ${ }^{18}$ Existen diversos análisis en los que se señala que el hecho de que se plantee una posible objeción democrática, no quiere decir que no sea posible justificar el control jurisdiccional de la ley. Esta premisa ha dado lugar a construcciones teóricas que buscan ofrecer razones para responder a la dificultad contramayoritaria. De hecho, entre los argumentos que van en esta dirección, uno evidentemente importante es el que sostiene que el control jurisdiccional de la ley es perfectamente democrático desde el momento en que las constitu-

17 Nagel, Robert F., Constitutional cultures: The mentality and consequences of judicial review, Los Angeles, University of California Press, 1989, p. 23. Para Nagel "If the practices that give meaning to the Constitution are acknowledged, the capacity of the nonjudicial institutions to sustain constitutional standards need not be viewed so pessimistically". Es más, para dar fuerza a sus planteamientos, este autor refiere, como ejemplo, que en los Estados Unidos "It was [...] the public opinion and a censure vote in the legislative branch, not judicial intervention, that stopped Senator Joseph McCarthy's threat to first amendment freedoms. It was public indignation at Franklin Roosevelt's court-packing plan that protected the independence of the judiciary".

18 La idea de hablar de una "oportunidad contramayoritaria" es tomada de Ferejohn, John y Pasquale Pasquino, op. cit., passim. 
ciones, aprobadas democráticamente y en consecuencia muy probablemente también democráticas en sus contenidos, muchas veces establecen explícitamente ese control. ${ }^{19}$

A este planteamiento se le da mayor fuerza a través de argumentos relacionados con la legitimidad democrática de la Constitución. De acuerdo con esta idea, el control jurisdiccional de constitucionalidad es compatible con la democracia pues "cuando los jueces ponen en vigor la Constitución descalificando una ley o un decreto no están oponiéndose a la voluntad mayoritaria, sino que -contrariamente- están honrando la soberanía popular expresada en esa misma Constitución”. De esta manera, lo que los órganos de control estarían haciendo es "darle eficacia a una voluntad mayoritaria (la del Constituyente) de nivel superior a la del legislador ordinario o el administrador". ${ }^{20}$

19 Ferreres Comella, Víctor, op. cit., pp. 47-49. Ferreres señala que incluso en aquellos sistemas en que los mecanismos de control jurisdiccional de la ley no se encuentran contemplados de manera explícita en el texto constitucional, el contenido democrático del control jurisdiccional podría seguirse sosteniendo en la medida que una institución será o no democrática desde el punto de vista de su estructura objetiva según cuáles sean sus características, y no por el hecho de que se cree a través de un proceso democrático. Este punto es importante en la práctica pues "si los miembros de una comunidad política comparten convicciones democráticas, tratarán de interpretar y reformar sus instituciones de acuerdo con los principios democráticos; en consecuencia, si consideran que determinada institución sólo se ajusta a los principios democráticos si se interpreta de determinada manera, tendrán una razón de mucho peso para interpretar la institución de esa manera [...] En este sentido, [por ejemplo,] muchas de las propuestas que se hacen en los Estados Unidos para fijar cuál debe ser el papel del juez constitucional en un régimen democrático se mantendrían inalteradas si mañana se reformara la Constitución americana y se declarara explícitamente que los jueces tienen el poder de controlar la constitucionalidad de la ley".

20 Álvarez Álvarez, Fernando D., op. cit., pp. 156-160. Este autor señala como otro de los argumentos para sostener el control jurisdiccional de la leyes la falta de una alternativa institucional razonable (de lege data) a este sistema. 
Un argumento que también suele ofrecerse frente a quienes impugnan por razones democráticas el control jurisdiccional de constitucionalidad es el de los derechos. Para muchos, pueden existir ciertas características antidemocráticas de este tipo de control; sin embargo, ocurre que el principio democrático no es el único con arreglo al cual se deberían diseñar las instituciones del Estado. Al lado de este principio se encuentra el de protección de los derechos que, se dice recurrentemente, debe prevalecer frente al principio democrático en caso de conflicto. ${ }^{21}$ Los argumentos basados en el tema de los derechos tratan de justificar la autoridad contramayoritaria de los órganos de control, señalando que ciertos derechos básicos son necesarios para alcanzar los compromisos con la libertad, la independencia o la igualdad contenidos en los textos constitucionales. ${ }^{22}$ Quienes hacen suyos estos argumentos asumen que debido a su formación y a su relativo aislamiento de la opinión pública (o por ambos motivos), los jueces disfrutan de ciertas ventajas institucionales sobre los legisladores respecto al proceso de descubrir, afirmar e implementar los derechos humanos. Por lo tanto, el control jurisdiccional de constitucionalidad se justifica por ser un medio necesario a través del cual se garantiza la protección de estos derechos. ${ }^{23}$

21 Ferreres Comella, Víctor, op. cit., p. 49.

22 Bassok, Or, op. cit., p. 344. Sobre este punto, y haciendo referencia a las democracias liberales, Michael J, Perry señala:"the foundational moral commitment of liberal democracy is to the true and full humanity of every human being -and, therefore, to the inviolability of every human beingwithout regard to race, sex, religion, etc. This commitment is axiomatic for liberal democracy [...] Moreover, this foundational commitment to the inviolability of every human being is a principal ground of liberal democracy's further commitment to certain basic rights, understood as human rights. Indeed, these two allied commitments - to the humanity/inviolability of every human being and to certain basic rights- are constitutive of liberal democracy; they are what make a democracy a 'liberal' democracy". Perry, Michael J., "Protecting human rights in a democracy: What role for the courts", Wake Forest Law Review, vol. 38, 2003, pp. 638-639.

23 Bassok, Or, op. cit., p. 345. 
A la defensa de los derechos se agrega otro argumento para dar fuerza a las virtudes democráticas del control jurisdiccional de constitucionalidad: la ampliación de su objeto al proceso político democrático. ${ }^{24}$ En este sentido, siguiendo a John Hart Ely ${ }^{25}$, se ha señalado que los procedimientos democráticos son el resultado de reglas que, a su vez, buscan asegurar la eficacia de determinadas precondiciones de la democracia. Entre ellas se alude constantemente al sufragio universal, la igualdad en las condiciones en que tiene lugar la participación; la libertad de expresión; los mecanismos para evitar las mayorías congeladas y las manipulaciones de los sistemas electorales, etc. De esta forma, el grado de cumplimiento de esas condiciones refleja la calidad democrática de una sociedad determinada, pues la "finalidad de las reglas del proceso democrático es justamente tratar de asegurar el mayor grado de cumplimiento de esas condiciones, de modo que las decisiones políticas que se adopten, básicamente a través de leyes, conduzcan a la concreción de principios morales, que permitan la plenitud de las personas". ${ }^{26}$

Un argumento más que se emplea para defender la valía del control jurisdiccional de constitucionalidad en los Estados democráticos es que éste cumple una función fundamental, particularmente cuando los poderes públicos que encarnan la eficacia de la acción

24 Álvarez Álvarez, Fernando D., op. cit., p. 160 ss.

25 Ely, John H., Democracy and distrust. A theory of judicial review, Cambridge, Harvard University Press, 1980.

26 Álvarez Álvarez, Fernando D., op. cit., p. 161. Para Álvarez "El juez viene a actuar como un árbitro en una competencia deportiva: no debe intervenir para indicar que alguno de los equipos debe jugar de tal o cual manera ni, mucho menos, participar en el juego, sino simplemente limitarse a actuar cuando alguno de los dos equipos pretende obtener ventajas abusivas (este enfoque puede compararse al que en materia económica se denomina orientación antitrust). En la relación 'justicia-política' ocurre lo mismo, de modo que los jueces no deben intervenir para la determinación acerca de cómo debe funcionar el mercado político o cuáles deben ser los resultados, [pero sí] deben velar para que dicho mercado no funcione inadecuadamente". 
de gobierno se exceden de sus marcos de actuación establecidos en la Constitución. De esta forma, como señala Fernando Álvarez, cuando alguno de los poderes actúa en detrimento de los derechos de los integrantes del cuerpo social o de las competencias que tienen asignadas, los órganos de control juegan el papel de moderadores del proceso político y se convierten en elementos esenciales en los equilibrios de poder al buscar entre los diversos órganos del Estado la cooperación y evitar la expansión de uno en perjuicio del otro. ${ }^{27}$

Álvarez señala también que la facultad de controlar la constitucionalidad a través de medios jurisdiccionales es un poder limitado. Para él, la primera de las limitaciones que encuentra esta facultad es el hecho de que los jueces sólo puedan emitir juicios de inconstitucionalidad de actos o normas emanados de los poderes públicos en el marco de un caso determinado. La autorrestricción judicial (self-restraint) es, por tanto, otro de los límites que se señalan como uno de los elementos que contribuyen a la legitimidad democrática de los órganos de control. Esta teoría, sostenida entre otros por Holmes, Frankfurter y Brandeis, está basada en el respeto a la autoridad del Congreso y del Presidente, así como en la llamada doctrina de la deferencia hacia los actos de dichos poderes. Como consecuencia de esta doctrina los jueces deben dejar a los poderes políticos la solución de los conflictos constitucionales. ${ }^{28}$

Entre los argumentos que pueden utilizarse para legitimar la actuación de los órganos jurisdiccionales de control se encuentra también la estructura del razonamiento práctico justificatorio de los jueces constitucionales. Este razonamiento parte de proposiciones normativas que resultan aceptables por sus propios méritos y con prescindencia de que hayan sido emitidas por determinada autoridad. En consecuencia:

27 Ibidem, p. 163.

28 Ibidem, p. 164 ss. 
Cuando el contenido de esas proposiciones es apropiado, ellas constituyen juicios o principios morales, y éstos, a la vez que son el punto de inicio del razonamiento justificatorio, integran muchas veces los contenidos constitucionales. Así, tanto un ideal de constitucionalismo vinculado con los derechos como con la participación democrática pueden conformar principios legitimantes de determinadas autoridades, y pueden integrar la Constitución normativa. Precisamente esta Constitución es la que puede servir de premisa mayor de un razonamiento justificatorio de la decisión inicial. Esto conduce a sostener que el control judicial de constitucionalidad es inevitable, ya que los jueces no pueden justificar sus decisiones en el aspecto fáctico de la sanción de la ley [...], sino que necesariamente deben fundamentar tales decisiones en principios morales que son aceptados por sus méritos intrínsecos -tales como los que integran la constitución en sentido normativo. ${ }^{29}$

Este argumento, por tanto, legitima la actuación de los jueces constitucionales desde un punto de vista lógico, pues el tipo de control que realizan, de gran amplitud, "les permite ponderar valorativamente la práctica jurídico-institucional vigente, además de legitimar, si fuera necesario, el trascender una ley para determinar si su aplicación, en el caso concreto, satisface o no las condiciones para la continuidad de tal práctica". ${ }^{30}$

Sobre la dificultad de enmendar los textos constitucionales como obstáculo para el ejercicio democrático, puede decirse, con Ferreres, que esta situación no implica que el principio de protección de los derechos esté por encima del principio democrático, sino que, como se ha señalado, dentro del propio principio democrático se establece una jerarquía entre la voluntad de una supermayoría y la voluntad de una mayoría simple. De esta forma, si "se insiste en que el principio de los derechos debería tener prevalencia frente al principio democrático, habrá que argumentar entonces por qué se da esa prevalencia"; sin embargo, si se dice, por el contrario,

29 Idem.

зо Idem. 
"que no debe haber una prevalencia absoluta, sino que hay que buscar un punto de equilibrio entre democracia y derechos, habrá que indagar entonces cuáles son los límites que debe respetar el juez constitucional para no alejarse de ese equilibrio". ${ }^{31}$

Por lo que se refiere a la objeción basada en la idea de que los jueces estén en posibilidad de imponer su punto de vista a los ciudadanos de manera regular y por largos periodos, podría responderse, como lo hacen Ferejohn y Pasquino, en el sentido de que es difícil que los órganos de control pretendan imponer una dictadura judicial a la sociedad. ${ }^{32}$ De hecho, Friedman expresa lo difícil que pueden ser las confrontaciones directas entre los ciudadanos y los órganos de control, al retomar las palabras de Shrewd, quien refiriéndose a los desacuerdos entre Roosevelt y la Suprema Corte de los Estados Unidos, señaló: "No appointive body of nine men can fly in the face of public opinion for too long without provoking an aswering attack". ${ }^{33}$ Retomando estos señalamientos, Pasquino y Ferejohn señalan que la Corte y la opinión pública raramente pueden estar alejadas por largos periodos, por lo menos en asuntos que son trascendentes para un grupo importante de personas; por el contrario, los argumentos que defienden tienden a converger en el tiempo. Sin embargo, estos autores señalan también que existen múltiples maneras en las que es posible que dicha convergencia se produzca, y cada una de ellas puede tener implicaciones diferentes para la democracia: "The Court's jurisprudence could change because some members change their views, possibly in response to felt political pressures, or because some members are replaced. But it is also possible that on some issues, public opinion moves toward the

31 Ferreres Comella, Víctor, op. cit., p. 49.

32 Ferejohn, John y Pasquale Pasquino, op. cit., p. 355.

33 Friedman, Barry, The will of the people. How public opinion has influenced the Supreme Court and shaped the meaning of the Constitution, Nueva York, Farrar, Straus and Giroux, 2009, p. 370. 
Court's favored position". ${ }^{34}$ Ahora bien, también es posible que en ciertos casos, tanto el punto de vista de los órganos de control como el de la opinión pública coevolucionen "deliberativamente" a través de una especie de diálogo en formas que tienden a acercarlos más que a alejarlos. En cuestiones controvertidas como el aborto, los derechos de los homosexuales o la eutanasia, esta evolución parece estar teniendo lugar cada vez con mayor fuerza. ${ }^{35}$

En cualquiera de estos casos, sin embargo, los órganos de control, más que detener el debate, generan condiciones para que ciertos temas controvertidos se coloquen en la agenda pública, lo que a su vez favorece la democracia. Es verdad que no siempre los temas tratados por quienes realizan funciones de control constitucional trascienden las salas en las que se toman las decisiones, pero cuan-

34 Ferejohn, John y Pasquale Pasquino, op. cit., p. 357.

35 Ibidem, p. 358. Pasquino y Ferejohn, al tratar la obra de Friedman, señalan que ésta presenta "an ideal-typical sequence: 'The Court rules. The public responds'. Over time, sometimes a long period, public opinion jells, and the Court comes into line with the considered views of the [...] public. Friedman tends to emphasize in his examples the second part of this process, in which the Court somehow does the conforming. He does not neglect the first stage -where the public (if it pays much attention) responds to a decision and argues and deliberates to arrive at its considered views on the matter. Rather, he puts less emphasis on this mechanism. And he certainly emphasizes that the public debate takes place in light of the actions of the Court, and is therefore likely subject to judicial influence to some extent. The emphasis on judicial conformity neglects the fact that in most cases, it was a decision by the Court that set the public debate going, and that the Court itself played a major role in laying out the terms under which public arguments are made and judged. The point is that the agenda is set and structured by an initial decision. Thus, if the Court later on revises the initial decision or even overturns it, the basic ideas and considerations were, in many cases, already implicit (or even explicit) in its earlier rulings. If this is right, it may not be accurate to think of the Court beign pulled along by public opinion (which is, in many cases, not very well developed). It may be more correct to view the Justices as deliberating through concrete cases that come before them, while the public, too, deliberates, and reflects on similar issues." 
do lo hacen, lo que se logra es inducir el debate público, por lo que bajo esta premisa, la naturaleza contramayoritara del control jurisdiccional, más que una dificultad puede ser una oportunidad para que las democracias se vuelvan más deliberativas. No son raros los casos en que los órganos de control han colocado temas importantes en la agenda política y los han mantenido ahí por largos periodos, estimulando la participación de grupos de interés, partidos políticos, instituciones y ciudadanos que reaccionan y tratan de encontrar o crear nuevas iniciativas que puedan superar el examen de los jueces constitucionales. Cuando esto sucede, los órganos de control generalmente delinean y dan forma al conflicto, exponiendo aspectos constitucionales que los actores políticos habían olvidado o a los que no se les había dado el valor que merecían. Los esfuerzos que los órganos controlantes realizan en esa dirección plantean escenarios adecuados para un subsecuente debate público en el que frecuentemente se revelan las divergencias políticas y los conflictos constitucionales que existen y que son importantes para la sociedad. El efecto de la actuación de los órganos de control es entonces, en muchas ocasiones, abrir la puerta a una amplia deliberación que provoca y activa a diversos grupos de la sociedad que esbozan o incluso definen determinados temas controvertidos desde el punto de vista constitucional o convencional, así como incluir esos temas con fuerza en el debate público hasta que en el ámbito político o institucional se tomen las decisiones necesarias para redefinir la forma en que serán tratados. ${ }^{36}$

Para sumar argumentos a la defensa de la legitimidad democrática de los órganos de control, se ha dicho también que muchas

36 Ibidem, p. 360. Sobre la apertura de este debate, Ferejohn y Pasquino han señalado que "Importantly, Court-induced deliberation has seldom been restricted to occupants of formal governmental institutions, nor to a narrow political or social elite, and certainly not to the judiciary or to Court itself. Rather, it seems to have been more or less regularly open to new voices and new forms of speech and action arising from the People Themselves (so to speak)". 
veces su actuación corresponde en mayor medida a las aspiraciones de la sociedad que las determinaciones tomadas por los órganos legislativos. Or Bassok señala al respecto que antes de que existieran mecanismos científicos para medir la opinión pública, se consideraba que las decisiones del Congreso eran las que la reflejaban y, en consecuencia, poner en duda la actuación de ese órgano representativo era ir en contra de la voluntad de la mayoría. No obstante lo anterior, Bassok evidencia que a partir de la aparición de mecanismos eficaces para medir la opinión pública, esta situación ha cambiado ya que no son raras las ocasiones en que la sociedad tiene desencuentros con el órgano legislativo y sus opiniones respecto a temas diversos se alejan de las de sus representantes. Por ello refiere que actualmente podría hablarse en muchas ocasiones no de una dificultad contramayoritaria, sino "contrarrepresentativa". ${ }^{37}$

Partiendo de estas premisas, Or Bassok realiza un análisis conceptual en el que identifica dos tipos de contramayoritarismo. En su sentido tradicional, la dificultad contramayoritaria estaría relacionada con lo complejo que resulta aceptar que el principio democrático de la regla de mayoría pueda ser compatible con el hecho de que jueces que difícilmente se sujetan a responsabilidad por su actuación, invaliden la legislación aprobada por representantes que son responsables ante el electorado. Dado que los poderes que son elegidos a través de elecciones, si pretenden permanecer en el gobierno, deben seguir el pulso de la sociedad y adoptar las políticas señaladas por los ciudadanos, el que un pequeño número de jueces - cuya actuación no está determinada por la presión popular y difícilmente pueden ser responsables ante ella- tenga en sus manos el destino de la legislación aprobada por órganos representativos es algo criticable. ${ }^{38}$ Por su parte, la dificultad contramayoritaria en sentido literal tomaría en cuenta la forma en que la Corte responde

\footnotetext{
37 Bassok, Or, op. cit., pp. 337-339.

38 Ibidem, pp. 340-343.
} 
a la opinión pública. Para autores como Nathaniel Persily, si los órganos de control reflejan la opinión pública en sus decisiones, entonces sus decisiones no podrían ser descritas como contramayoritarias. ${ }^{39}$ De hecho, Persily ha señalado que:

Although the distinctive feature of the judicial role and position is its supposed insulation from the pressures of public opinion, in several recent cases the courts and litigants have put forth public opinion polls as evidence supporting a particular constitutional interpretation. When used in this way, appels to majoritarian sentiment (whether measurable or not) often justify a mode of constitutional argument that views the Constitution as a "living document" and judges role as helping the Constitution keep up with the times. ${ }^{40}$

Estas dos perspectivas sobre la dificultad contramayoritaria que encuentran puntos de contacto al dar un importante valor a la opinión de la mayoría, son también distintos el uno del otro. Cuando, de acuerdo con las encuestas de opinión, el público apoya la declaración de inconstitucionalidad de una ley que llevan a cabo los órganos de control, su actuación no podría considerarse contramayoritaria en sentido literal; sin embargo, seguiría teniendo este carácter en un sentido tradicional debido a que una institución que no es responsable frente al electorado deja sin efectos una norma que fue aprobada por un órgano cuya actuación sí se encuentra bajo el escrutinio directo de los electores. De esta forma, los órganos que tienen asignadas labores de control pueden actuar en forma contramayoritaria en el sentido tradicional del término siempre que

39 Para este autor, "the degree of difficulty presented by the Court's countermajoritarian status can be assessed by how out of step its decisions are with public opinion in its decisions, then whatever other problems it might have, it could not be described as countermajoritarian". Persily, Nathaniel, "Introduction", en Persily, Nathaniel, Jack Citrin y Patrick J. Egan (eds.), Public opinion and constitutional controversy, Nueva York, Oxford University Press, 2008, p. 5.

40 Idem. 
invalidan la decisión que toman órganos elegidos por los ciudadanos y no sólo - como podría implicar la definición de contramayoritarismo en sentido literal- cuando dejan sin efecto una ley que goza de una opinión favorable entre los integrantes de la sociedad. De la misma manera, un órgano de control jurisdiccional puede disfrutar del favor de la opinión pública y, consecuentemente, tenerse como una "institución mayoritaria", pero seguiría considerándose como contramayoritaria para una democracia desde el punto de vista tradicional. ${ }^{41}$ Sin embargo, debe considerarse también que:

the Court may enjoy durable majoritarian support as an institution even while deciding some cases in a [countermajoritarian (CM)] fashion in the literal sense. The public may disagree with certain decisions making them $\mathrm{CM}$ in the literal sense. However, the more durable public support for the Court as an institution can trascend such reactions to the Court's concrete performance and remain unscathed. Hence, the Court may be a majoritarian institution in the literal sense while deciding some cases against public opinion i.e., deciding literally in a CM fashion. ${ }^{42}$

No obstante lo anterior, como se ha referido, este hecho no debe entenderse en el sentido de que los órganos de control pueden actuar en contra de la opinión pública durante mucho tiempo, pues debido a que éstos no obtienen su legitimidad del voto de los ciudadanos, deben encontrarla en su actuación y, por tanto, hasta cierto punto, en la aceptación que ésta logre por parte de los integrantes de la sociedad. En este punto es necesario distinguir entre el respaldo difuso y el respaldo específico que se da a los órganos de control jurisdiccional. Como señalan Caldeira y Gibson, cada ciudadano puede en determinado momento estar en desacuerdo con las políticas que llevan a cabo esos órganos de control, desconfiar de sus integrantes o criticar sus procedimientos. Normalmente, sin embargo, dichas actitudes no se traducen en una disminución del respaldo a la insti-

41 Bassok, Ork, op. cit., p. 342 ss.

42

Idem. 
tución. De hecho, un ciudadano puede estar en desacuerdo con lo que una institución hace, pero a pesar de ello continuar pensando en ella como el órgano legitimado para tomar ciertas decisiones. Bajo estas premisas, el "respaldo específico" se presenta a través de ciertas actitudes hacia una institución basadas en el cumplimiento por parte de ella de las demandas sobre un determinado tipo de políticas o acciones; mientras que el "respaldo difuso" se relaciona con actitudes favorables o con la buena disposición hacia la institución, que ayudan a los integrantes de la sociedad a tolerar decisiones a las que se oponen o a aceptar los efectos de aquello que consideran perjudicial para sus intereses. ${ }^{43}$

El respaldo difuso puede considerarse, consecuentemente, el elemento que los órganos jurisdiccionales de control necesitan para tomar decisiones contramayoritarias y poder seguir gozando de la legitimidad que requieren en su actuar. Y este respaldo, aunque pudiera parecer paradójico, lo obtienen en la medida en que rara vez obstruyen importantes políticas compartidas por las mayorías nacionales. En los Estados Unidos, por ejemplo, diversos estudios han demostrado que existe una correlación entre la opinión de los ciudadanos medida por las encuestas y las decisiones de la Corte en los asuntos que tienen un sobresaliente tratamiento público. Dichos estudios empíricos han evidenciado que a lo largo del tiempo las resoluciones de los órganos jurisdiccionales de control tienden a reflejar la opinión de la mayoría en lugar de frustrarla o confrontarla. ${ }^{44}$

43 Caldeira, Gregory A. y James L. Gibson, "The etiology of public support for the Supreme Court", American Fournal of Political Science, vol. 36, núm. 3, 1992, p.637.

44 Bassok, Or, op. cit., p. 351. Jeffrey Rosen comparte esta opinión al señalar que "Beginning with Robert Dahl in the 1950s, political scientists have argued that the Supreme Court throughout its history has tended to follow national opinion rather than challenging it". Véase la introducción a The most democratic branch: How the Courts serve America, Nueva York, Oxford University Press, 2006. 
De hecho, existen diversos argumentos a través de los cuales se trata de explicar la relación que hay entre el comportamiento de los órganos jurisdiccionales de control y la opinión pública. El primero de ellos está relacionado con la forma en que se nombra a los jueces constitucionales, pues dado que generalmente en este proceso participan órganos representativos, las mayorías que son necesarias para la designación asegurarían cierta sincronía entre la actuación de las legislaturas y las decisiones de los integrantes de los órganos de control. ${ }^{45}$ Este argumento, sin embargo, podría ser cuestionado en los sistemas en que el proceso de designación o los periodos de duración del cargo evitan la referida sincronización entre la participación del órgano representativo en los nombramientos y quienes a través de la jurisdicción realizan labores de control.

El segundo argumento para explicar la correlación referida es que los órganos de control se comportan como jugadores estratégicos en el entramado institucional y modifican su actuación tomando en cuenta la opinión pública y el comportamiento de otros jugadores políticos mayoritarios, con el fin de proteger el respaldo público del que gozan (su legitimidad sociológica), así como su eficacia política. De acuerdo con estos planteamientos teóricos, una de las preocupaciones de los jueces constitucionales es que la disminución del respaldo que tienen por parte de la opinión pública pueda implicar una pobre implementación de sus decisiones, provoque reacciones negativas o pueda tener otro tipo de consecuencias. ${ }^{46}$ Por ende, para evitar este declive en su actuación, los órganos de control responden, hasta cierto punto, a la opinión pública. ${ }^{47}$

\footnotetext{
45 Bassok, Or, op. cit., pp. 352-354.

46 Ibidem, p. 355.
}

47 Lawrence Baum y Neil Devins señalan al respecto: "Scholars who perceive a linkage between the Court and the public emphasize what they see as the Justices' concern with the Court's legitimacy. Other possible sources of influence for public opinion (most notably, the Justices' concern with their individual popularity) are tipically given no weight. In this view, 
El modelo actitudinal, que se ha empleado también para justificar la relación tratada sugiere, por su parte, que los jueces constitucionales tomen ciertas decisiones basándose primeramente en sus ideologías, que se delinean a través de factores similares a aquellos que dan forma a las preferencias ideológicas de la opinión pública y sus representantes. En la medida que los jueces constitucionales compartan las preferencias ideológicas del público, consecuentemente, tenderán a reivindicar la opinión pública incluso sin intentar hacerlo. ${ }^{48}$

Algunos autores señalan como un elemento más para explicar la relación entre las decisiones que toman los órganos de control y la opinión pública, las reacciones que ésta pueda tener ante la actuación de los jueces de control. Si entre la sociedad se da una desaprobación intensa y sostenida por parte de los ciudadanos respecto a una decisión jurisdiccional y ésta se acompaña por actitudes agresivas para oponerse a dicha decisión e intentar quitarle su fuerza legal, los órganos de control pueden intentar alinearse con la opinión de los disidentes. Por lo tanto, las movilizaciones sociales se constituyen en otro mecanismo que asegura que los órganos jurisdiccionales sean responsables de cierta forma ante la opinión pública. ${ }^{49}$ De hecho, Friedman al tratar teóricamente el "matrimonio" que existe entre la Suprema Corte de los Estados Unidos y la opinión pública, ha señalado que a pesar de que aquélla siempre ha estado atenta al pulso de la sociedad, sólo recientemente ambas han entendido cómo interactuar de manera efectiva. Para él:

the Justices respond to public views because they are concerned with the Court's efficacy as a maker of legal policy. Lacking concrete sources of power, the Court depends on its public legitimacy". Estos planteamientos se encuentran en "Why the Supreme Court cares about elites, not the American people", The Georgetown Law fournal, vol. 98, 2010, p. 1547.

48 Bassok, Or, op. cit., p. 355.

49 Ibidem, p. 356. 
Now that the justices and the public understand how things work, the system tends to rest in a relatively quiet equilibrium. Political scientists call this anticipated reaction. The justices don't actually have to get into trouble before retribution occurs: they can sense trouble and avoid it. The people do not actually have to discipline the justices; if they simply raise a finger, the Court seems to get the message. ${ }^{50}$

Este mecanismo de equilibrio a través del cual Friedman explica la sincronía que existe entre los órganos jurisdiccionales de control y la opinión pública, enfatiza la anticipación que se logra a través del "temor" a una reacción pública. ${ }^{51}$

Además de estos argumentos, se ha señalado que en ciertas áreas del derecho la opinión pública, cuando es fuerte, se incorpora al discurso legal como una fuente de legitimación de la interpretación constitucional, incluso sin pasar por el proceso democrático formal y sin que exista la amenaza de una reacción por parte de la opinión de los integrantes de la sociedad. En estas áreas la única cuestión relevante es si dicha opinión es lo suficientemente fuerte. Consecuentemente, la línea que separa a la opinión pública del lenguaje legal desaparece completamente pues aquélla se incorpora a éste. Una de las implicaciones que tiene esta alineación entre los contenidos de las normas constitucionales y una arraigada opinión pública es, por tanto, la disolución de la dificultad contramayoritaria en su sentido literal. ${ }^{52}$

Por otra parte, en lo que se refiere al señalamiento de que a través de la actuación de los jueces constitucionales se coarta al pueblo soberano, en ciertas circunstancias extremas, la posibilidad de desatender el orden jurídico para preservar la moral representada por su propia comunidad política, debe decirse que los órganos de control han sido considerados recurrentemente como los guardianes

50 Friedman, Barry, The will of the people, op. cit., pp. 376 ss.

51 Idem.

52 Bassok, Or, op. cit., pp. 357-358. 
del principio de composición del cuerpo social. Esto, pues tienen como función mantenerlo inalterado, es decir, preservar las condiciones que hacen su poder soberano, igual a sí mismo en todos los instantes del tiempo. De esta manera, si los órganos jurisdiccionales de control se oponen al pueblo, es simplemente con el fin de evitarle ponerse en contradicción consigo mismo. ${ }^{53}$ Además, es necesario tomar en cuenta que incluso si en circunstancias de normalidad política los órganos de control pueden dejar sin efecto las decisiones de los poderes ejecutivo y legislativo, en aquellas situaciones críticas en las que lo que está en juego es la propia comunidad política, los poderes electos democráticamente buscan coordinarse con los órganos jurisdiccionales para atender estas situaciones o simplemente los ignoran. Y es que es obvio que en momentos de crisis, los controlantes no pueden realizar juicios que impidan la autodefensa del sistema. ${ }^{54}$ En este sentido, Gowder menciona que:

Not only do judges tend to feel no particular desire to turn their constituions into suicide pacts, theh have self-interested incentives not to do so, since their power and prestige also depends on the continuing existence of the democratic legal systems from which they draw authority. In those rare cases when the judiciary has tried to stand against the other branches in emergencies, it has found itself powerless. ${ }^{55}$

Finalmente, por lo que hace a los niveles de fiscalización a los que pueden estar sujetos los órganos de control, no debe olvidarse que el pueblo y sus representantes (elegidos democráticamente) han tenido a lo largo del tiempo la posibilidad de presionar bajo ciertas circunstancias a los órganos de control y lo han hecho en numerosas ocasiones. La fiscalización de estos órganos de control por parte de la voluntad popular puede consecuentemente expresarse aun cuan-

53 Álvarez Álvarez, Fernando D., op. cit., p. 163.

54 Gowder, Paul, op. cit., p. 15.

55 Ibidem, p. 16. 
do los jueces constitucionales gocen de cierta libertad en su actuar, porque es el propio pueblo el que les ha dado dicha libertad. El poder jurisdiccional para controlar normas y actos de autoridad deriva entonces de la dispensa que le otorga la sociedad a los órganos de control. ${ }^{56} \mathrm{Y}$ no debe olvidarse, como señalara Bickel, que estas posibilidades de actuación derivan de las capaciadades que tienen para atender cuestiones de principio que ni las legislaturas ni el Ejecutivo poseen. ${ }^{57}$ Además, debe recordarse también que existen mecanismos como el impeachment, a través de los cuales los jueces constitucionales pueden ser sujetos de control en casos extremos, cuando los poderes políticos del Estado consideran que éstos han incurrido en responsabilidad por un mal desempeño en el ejercicio de sus funciones. Estos mecanismos deben emplearse con cautela cuando se dirigen a los órganos de control, pues pueden implicar la búsqueda de la conquista total del poder por mayorías accidentales en el poder legislativo; pero a pesar de este riesgo, no cabe duda de que figuras como el impeachment se presentan como instrumentos de control (extremos, por cierto) que los representantes del pueblo tienen sobre los órganos jurisdiccionales que revisan la constitucionalidad de leyes y actos de autoridad. ${ }^{58}$

56 Friedman, Barry, The will of the people, op. cit., p. 370.

57 Para este autor "Judges have, or should have, the leisure, the training, and the insulation to follow the ways of the scholar in pursuing the ends of government. This is crucial in sorting out the enduring values of a society, and it is not something that instituions can do well occasionally, while operating for the most part with a different set of gears [...] The courts are concerned with the flesh and bood of an actual case. This tends to modify, perhaps to lengthen, everyone's view. It also provides an extremely salutary proving ground for all abstractions; it is conducive, in a prhase of Holmes, to thinking things, not words, and thus to the evolution of principle by a process that tests as it creates". Bickel, Alexander, op. cit., pp. 25-26.

58 Álvarez Álvarez, Fernando D., op. cit., p. 163. 
Este recorrido a través de las objeciones a la actuación contramayoritaria de los órganos de control, demuestra que cada una de ellas ha encontrado una respuesta en la doctrina que refleja oportunidades en los sistemas democráticos. Y es que el control de constitucionalidad realizado por órganos jurisdiccionales non sólo protege los contenidos sustanciales de la democracia, sino que trata también de asegurar las condiciones que reflejan la calidad democrática de una sociedad determinada, ponderando valorativamente la práctica jurídico-institucional que en ella se desarrolla.

IV. A mANera de CONCLUSIONES

Las objeciones a la actuación contramayoritaria de los órganos de control pocas veces encuentran cabida en las sociedades democráticas modernas. Al final del día, si los órganos jurisdiccionales, como se señalaba en El Federalista, no cuentan ni con la bolsa ni con la espada, sólo les queda la confianza de la sociedad-que se basa en el prestigio del que gozan- para permanecer como una pieza fundamental en el entramado institucional existente en los actuales Estados constitucionales. Es por ello que como actores que tienen a su cargo una de las funciones fundamentales en las democracias (servir como medio de coordinación a fin de que el pueblo cuente con canales institucionales para responder ante conductas inconstitucionales) deben gozar, al menos por regla general, del apoyo del cuerpo social. Este presupuesto es fundamental para los órganos de control, pues éstos son instituciones especialmente vulnerables al carecer de una conección electoral que les provea legitimidad y tener en ocasiones que actuar contra la opinión pública para defender ciertos principios y valores contenidos en el texto constitucional. Sin embargo, su actuación más que verse como una dificultad en los sistemas democráticos, debe concebirse como una oportunidad, pues al usar medidas contramayoritarias para detener u obstaculizar los deseos de las mayorías cuando éstos son 
contrarios a la Constitución, abren un espacio importante para la deliberación pública, incluyendo en ella nuevos puntos de vista que muchas veces escapan a las determinaciones gubernamentales y se sitúan fuera de las fronteras de lo políticamente correcto. Ahora, si bien es cierto que la forma en que los órganos de control cumplen con sus funciones favorece los procesos democráticos, no deben perderse de vista los riesgos de una excesiva politización de la justicia o de una perjudicial judicialización de la política. El reto entonces es encontrar un punto en el que sea posible la convergencia de los postulados propios del Estado constitucional con los deseos de la mayoría y, para ello, contar con un sistema de control bien estructurado se torna un elemento fundamental en los países democráticos. 